\title{
ERRATUM
}

\section{Erratum to: Biomineralized and chemically synthesized magnetic nanoparticles: A contrast}

\author{
Tanya NANDA ${ }^{a}$ ), Ankita RATHORE ${ }^{\text {b ) }}$, and Deepika SHARMA (ه) \\ Institute of Nano Science and Technology, Habitat Centre, Sector 64, Mohali 160062, India
}

(C) Higher Education Press 2021

Erratum to: Front. Mater. Sci. 2020, 14(4): 387-401

DOI: $10.1007 / \mathrm{s} 11706-020-0531-7$

Page 391, the caption of Fig. 3 "Biomineralization and role of magnetosome membrane proteins in synthesis of BM crystals in MTB (Magnetosome proteins: MamR, MamS, MamT, MamP, MamD, MamF, MamG, MamE (protease dependent), MamC, MamM, MamN, MamO, MamK, MamJ, MamE, MamI, MamL, MamQ, MamB)." should read "Biomineralization and role of magnetosome membrane proteins in synthesis of BM crystals in MTB (Magnetosome proteins: MamR, MamS, MamT, MamP, MamD, MamF, MamG, MamE (protease dependent), MamC, MamM, MamN, MamO, MamK, MamJ, MamE, MamI, MamL, MamQ, MamB). Reproduced with permission of PNAS from the reference as follows: Dorothée Murat, Anna Quinlan, Hojatollah Vali, Arash Komeili. Comprehensive genetic dissection of the magnetosome gene island reveals the step-wise assembly of a prokaryotic organelle. PNAS, 2010, 107(12): 5593-5598.”

The authors apologize for any confusion.

Received December 12, 2020

E-mail: deepika@inst.ac.in

a) Present address: School of Biological and Health Systems Engineering, Ira A. Fulton Schools of Engineering, Arizona State University, Tempe, AZ 85281, USA

b) Present address: Academy of Scientific and Innovative Research (AcSir), CSIR-National Institute of Science Communication and Information Resources (NISCAIR), New Delhi 110067, India 